\title{
Printing Paper as a Reflector with Idealized Properties: How to Link the Paper Industry and Printing Art
}

\author{
Dmitry A. Tarasov ${ }^{1, \mathrm{a})}$, Andrey G. Tyagunov ${ }^{1, \mathrm{~b})}$, Oleg B. Milder ${ }^{1, \mathrm{c})}$ \\ ${ }^{1}$ Ural Federal University, Mira str., 19, Ekaterinburg, RUSSIA 620002 \\ ${ }^{a)}$ Corresponding author: datarasov@yandex.ru \\ b)adi8@yandex.ru \\ c)milder@mail.ru
}

\begin{abstract}
Whiteness is one of the most important characteristics of a printing paper. There is a stable relationship between the printed and optical properties of the paper. At the same time, the understanding of whiteness in the papermaking and printing industries is significantly different. Such miscommunication negatively affects the quality of printed products, since the choice of a suitable paper (or cardboard) is difficult to a certain extent. One of the ways to get out of this situation is to create a simple and clear computational model of the white paper, which would not depend on any physical standards. The model would help when conducting the input control in printing plants and would allow one to establish communication between industries. This work is devoted to the description of such a model.
\end{abstract}

Keywords: Whiteness, Brightness, Paper, Shade, Model

\section{INTRODUCTION}

Paper is a material with a weight of square meter up to $250 \mathrm{~g}$ consisting mainly of bonded and intertwined natural fibers. Additional strength and printing characteristics of each paper are achieved through the introduction of filling agents, mineral fillers, chemical and natural fibers, pigments and dyes [1]. Paper is the most important raw materials used in the print industry, publishing, and media industry.

Cellulose is known as the most significant fiber source for paper [2]. Cellulose is a semi-finished fibrous product obtained by boiling vegetable raw materials with chemical solutions, as a result of which the most non-cellulose components (lignin, hemicellulose, extractives) are removed. These semi-finished products have low whiteness that is assessed by comparing it with the standard barium sulfate, the whiteness of which is taken to be $100 \%$. This approach is used in the papermaking industry. Here, the whiteness of unbleached cellulose varies between $20-65 \%$, $23-28 \%$ for the sulphate pulp (brown color), and $60-70 \%$ for the sulphite one (gray color). That is why these semifinished products with such whiteness are unsuitable for chemical processing and production of white paper. Bleached cellulose (sulphite, bisulphite, sulphate) is a chemically bleached pulp. The purpose of the bleaching process is to give a high and stable whiteness to the fiber semi-finished product. Until now, the main bleaching agents are chlorine and its compounds (hypochlorites, chlorine dioxide). This is due to the high selective destructive effect of chlorine on lignin and other coloring agents. As a basis for the manufacture of paper, the cellulose plays an important role in terms of strength parameters and in terms of the optical characteristics of paper.

Most papers contain materials, so-called fillers, which are introduced to impart certain properties to the produced paper. The fillers can be finely dispersed relatively insoluble inorganic materials or minerals. The most common are clay, titanium dioxide, and calcium carbonate, which are combined into a composition during the paper making process to form a sheet. The purpose of the fillers is to change the characteristics of the finished paper, such as opacity, brightness, printing properties, texture, and weight. The fillers are also used to give softness, reduce bulkiness, increase smoothness, make paper more evenly perceiving printing ink, and give greater dimensional stability. The main reasons for introduction of the fillers in printing paper are increased opacity and brightness, reduced ink penetration, and decrease in the stiffness of the fibers. The fillers for papermaking should have high

International Conference of Numerical Analysis and Applied Mathematics ICNAAM 2019

AIP Conf. Proc. 2293, 140017-1-140017-4; https://doi.org/10.1063/5.0026743

Published by AIP Publishing. 978-0-7354-4025-8/\$30.00

140017-1 
brightness, good light-scattering properties to increase the opacity, and the absence of abrasiveness and chemical inertness. Aluminum hydroxide, talc, calcium sulfate, barium sulfate, natural or synthetic silicates, or silicate pigments, and zinc pigments are also used as fillers.

Printing paper plays a key role in the quality of finished printed products. When perceiving the quality of the finished product, the most important property of paper is its whiteness. There are thousand of paper grades, which manufacturers often provide with conflicting technical information. For example, the "whiteness" parameter might be specified in accordance with two different international standards, which makes it difficult for the consumer to choose the materials to be purchased. An important problem for the printing industry is the change in whiteness from batch to batch, while manufacturers specify the same value in the accompanying product documentation.

It is necessary that one has some convenient method to determine the whiteness of printing paper. The method should use the existing measuring base and a model of reflecting the influence of the mineral composition of the paper on its whiteness. These would facilitate the incoming quality control of the intrant raw materials and, thus, to provide the positive effect on the quality of the output product.

Whiteness is a complex property of the visual sensation, which characterizes the degree of the subject's approaching to the white color according to the strength of its increased brightness, high dispersibility, and minimum color shade [3-5]. Whiteness is one of the most important characteristics of the printing paper. The literature has repeatedly shown that there is a stable relationship between the printed and optical properties of the paper (whiteness, opacity, shade, etc.). The optical characteristics of paper depend on the nature, amount, and ratio of ingredients in the paper composition, on the uniformity of their distribution over the sheet thickness, and many other technological factors. The whiteness and tint of paper are responsible for the visual contrast and the quality of reproduction of text and illustrations in the printing reproduction. In [6], it was shown that the text contrast is one of the main parameters affecting the legibility of the text, its understanding, and memorization. This is especially important for children and inexperienced readers who only master the skills of reading. Thus, the control of the optical characteristics of the paper in publishing is one of the key points determining the quality of published products. At the same time, there were few studies of the effect of whiteness and other optical characteristics of paper on reading and perception of the printed information.

The paper manufacturers indicate the value of paper whiteness that is determined by one of the standard methods. According to the ISO $2470-77$ or the ISO-brightness method [3, 4] determines the integral (i.e. without spectral information) indicator of the whiteness of the printing paper and shows how the light is diffusely reflected within the visible spectrum in the "blue" areas with an effective wavelength of $457 \mathrm{~nm}$. Measurement of whiteness by this method is carried out using photometers (spectrometers) by comparing with a reference sample. Such sample uses a set of standard samples of barium sulfate tablets, magnesium oxide, titanium dioxide, or a plate of reflective neutral glass. A priori, it is assumed that the paper reflects the light according to the Lambert's law, i.e. in the diffuse way. This method only shows how much more or less the light in the visible band of the spectrum of this particular sample is able to reflect in comparison with the standard sample. In accordance with this standard, the whiteness may exceed $100 \%$, however, this only means that the integral reflection coefficient of the test sample exceeds the reflection coefficient of the standard one. Moreover, without specifying the standard, this information does not make sense.

According to the methodology of the International Commission on Illumination (CIE), the diffuse energy brightness coefficient is measured in the full visible spectral range under illumination from various light sources and different types of the observer (ISO 11475 [7] for the natural daylight, ISO 11476 [8] for the artificial lighting). The method is based on the one described in the GOST 30116-94 [9] and allows one to estimate the degree of whiteness of a sample containing optically bleaching agents and elements of a tint dye. However, it has several disadvantages. For example, the measuring sample should have a high degree of whiteness.

Printing art industry operates the paper whiteness as a value of particular CIE Lab color coordinates that are described in the ISO 12647 standards series [10]. Hence, the paper whiteness is assessed by three different inconsistent ways. This work is an attempt to build the model of the paper with idealized spectrum that might act as an independent and universal paper whiteness standard.

\section{MODEL DESCRIPTION}

Comparison of a large number of similar reflection spectra of paper samples is not applicable as a convenient method. We propose using the mathematical model IP (idealized paper) as a standard. The model describes an idealized reflecting surface with a smooth reflection spectrum that meets some certain criteria. As a quantitative 
criterion, we apply the color coordinates that meet the requirements of the ISO 12647-2, viz. CIE Lab 95/0/-2, illuminator D50. Application of the IP model to real samples is provided due to a local maximum in the violet region of the spectrum, corresponding to a small number of fluorescent additives used in the paper production.

As a basis of the model, the measured bleached pulp spectrum (see Fig. 1, line 3) was used, which is well described by the increasing exponential of the form $\rho=A * \exp (\lambda / t)+\rho_{0}$. This relation refers the reflectance coefficient $\rho$ at the wavelength $\lambda$ and the distribution parameters $\mathrm{A}, \mathrm{t}$, and $\lambda_{0}$ that require fitting. To simulate a fluorescent additive, we use a bell-shaped function, which relates the reflectance coefficient $\rho$ at the wavelength $\lambda$ and the distribution parameters $\lambda_{v}, \delta \lambda$, and $\beta$, which require selection. The function is shown in (1). The IP spectrum is the sum of these two spectra.

$$
\rho_{\mathrm{v}}=\frac{\beta}{1+\left(\frac{\lambda-\lambda_{\mathrm{v}}}{\delta \lambda}\right)^{2}}
$$

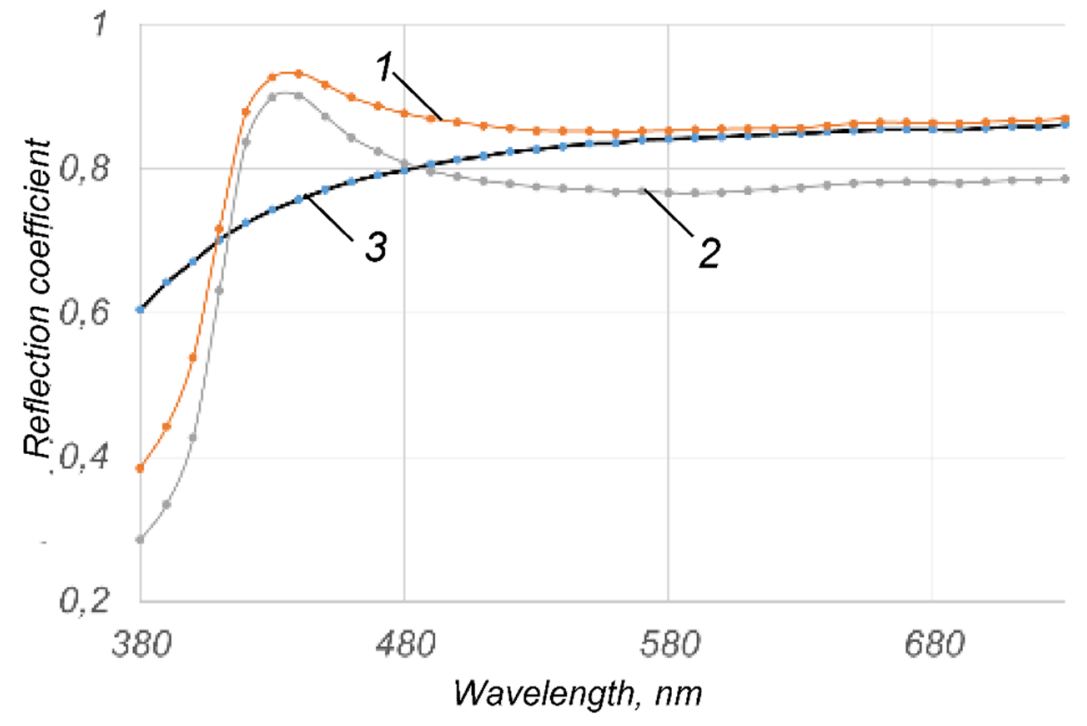

FIGURE 1. Examples of the reflection spectra of paper samples: coated paper (1); uncoated paper (2); bleached pulp (3).

The selection of the bleached pulp spectrum approximation curve was carried out in the Origin 9.2. The distribution parameters obtained in the approximation are: $\lambda_{0}=0.857 \pm 0.001 ; \mathrm{A}=-0.333 \pm 0.003 ; \mathrm{t}=-67 \pm 1$; coefficient of determination $\mathrm{R}^{2}=0,998$. Selection of the parameters of the fluorescent additive model was made in the MS Excel, the add-on "Search for solutions". When modeling, the parameters of the fluorescent additive model were changed so that the parameters of the total spectrum of the IP and its CIE Lab coordinates correspond to the requirements of the ISO 12647-2, i.e. CIE Lab 95/0/-2. The results of the parameters selection: $\beta=0.176 ; \lambda_{\mathrm{v}}=$ 413.875; $\delta \lambda=71.609$. The achieved color coordinates of the IP CIE Lab: $95.0 / 5^{*} 10^{-9} /-1.9$. This completely complies with ISO 12647-2 requirements. The synthesized IP spectrum is shown in Figure 2. The resulting IP model was used to conduct a visual comparative analysis of groups of paper samples by the applying spectra.

As a quantitative estimate of the difference between the reflection spectra of samples and IP suitable for industrial use, the standard deviation (RMS) was used [11]. Practically, as a production method for assessing the whiteness of paper, it is necessary to measure the reflection spectrum of a sample using a spectrophotometer and calculate the standard deviation from the IP. The lower the MSE value, the better the sample is. White paper is printing paper with the CIE Lab coordinates close to $95 / 0$ / -2 and a smooth reflection spectrum close to the IP for the visual (spectral overlap) or quantitative (calculation of standard deviation) estimates.

\section{CONCLUSION}

For a qualitative and quantitative comparison of a large number of paper samples among themselves, a reference that reflects certain paper characteristics in the entire spectral range is needed. These characteristics are the shape of the paper's reflection spectrum with a local maximum in the short-wavelength region and the CIE Lab coordinates $95 / 0 /-2$. This is a paper with an idealized spectrum (IP), whose mathematical model meets the specified 
characteristics. Comparison of paper samples using the IP might be carried out both qualitatively according to the nature of the placement of the spectra of the samples relative to the IP spectrum, and quantitatively by calculating the standard deviation of the monochromatic reflection coefficients of the samples and the IP. This creates the ability to produce quickly a large number of comparisons, as well as to automate this process.

Instead of different, unrelated definitions of paper whiteness, it is proposed using the one: "Paper whiteness is a complex property that characterizes the degree of paper sample approaching the standard (paper with idealized spectrum) according to the degree of smoothness of its reflection spectrum, the color coordinates are close to the standard values, high diffusing ability and minimum color shade". The printing and papermaking technology might develop the parameters of this standard. The proposed IP model makes it easy to recalculate the parameters of the standard to the new reference conditions.

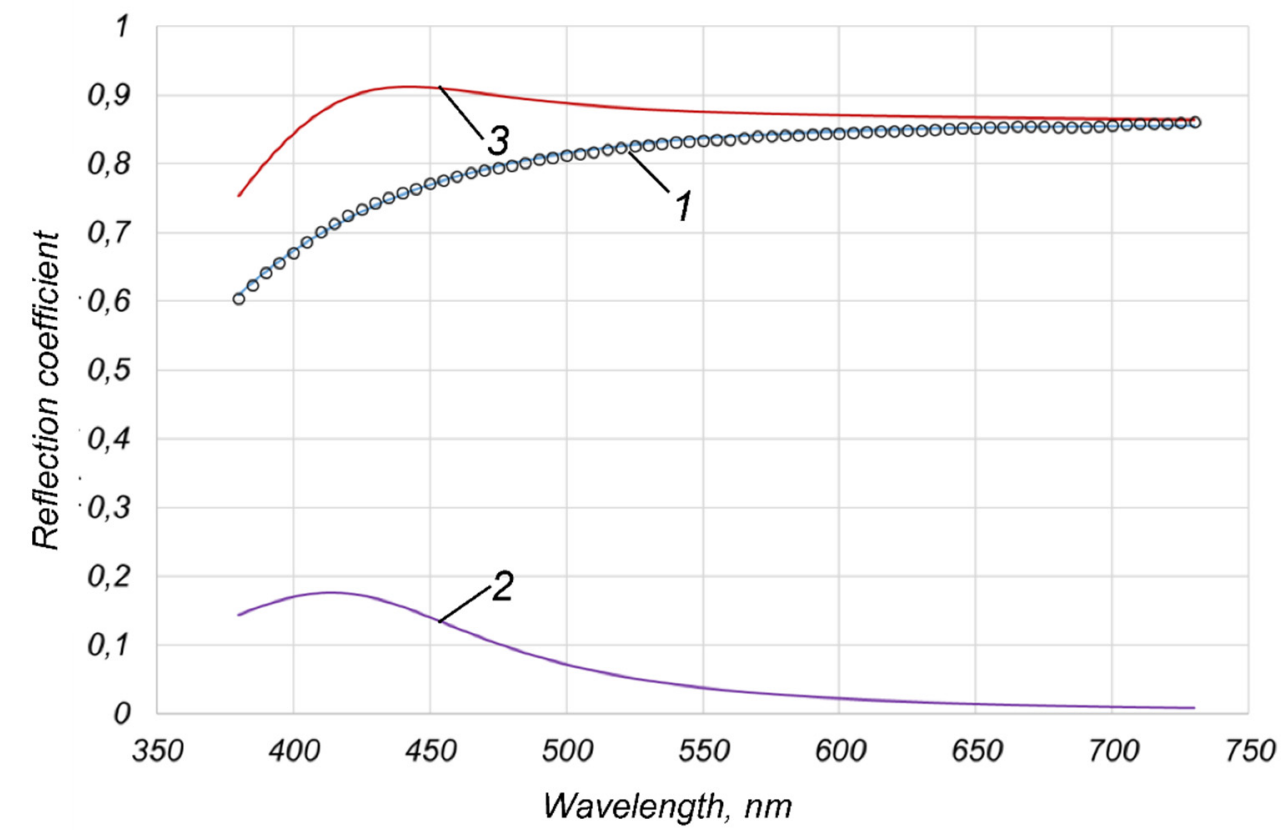

FIGURE 2. Synthesis of the IP spectrum: bleached pulp spectrum and its approximation curve (1), selected spectrum of the shortwave component (2), IP spectrum (3).

\section{REFERENCES}

1. ISO 15397:2014. Graphic technology - Communication of graphic paper properties

2. A.V. Vurasko, A.Ya. Ageev, and M.A. Ageev, Technology of production, processing and re-processing of paper and cardboard: tutorial. Ural Wood-Technical University publishing, Yekatetinburg, 272p. (2011) (in Russian).

3. ISO 2470-1, Paper, board and pulps - Measurement of diffuse blue reflectance factor - Part 1: Indoor daylight conditions (ISO brightness).

4. ISO 2470-2:2008, Paper, board and pulps - Measurement of diffuse blue reflectance factor — Part 2: Outdoor daylight conditions (D65 brightness).

5. N. Dubina, CompuArt, 11, 50-59 (2000). (In Russian).

6. D. Judd D and G. Wyszecki, Color in Business, Science, and Industry, Wiley-Interscience; 3rd ed, 576p. (1975)

7. ISO 11475, Paper and board — Determination of CIE whiteness, D65/10 degrees (outdoor daylight).

8. ISO 11476, Paper and board - Determination of CIE whiteness, C/2 degrees (indoor illumination conditions)

9. ISO 2469:2014 Paper, board and pulps -- Measurement of diffuse radiance factor (diffuse reflectance factor)

10. ISO 12647-7, Graphic technology - Process control for the production of half-tone colour separations, proof and production prints - Part 7: Proofing processes working directly from digital data

11. G. Ivchenko and Yu. Medvedev, Introduction in mathematical statistics, LKI, Moscow, 600p. (2010) (In Russian). 